\title{
Diabetes tipo 2. ¿Qué podemos esperar tras la adopción de los nuevos criterios diagnósticos? Incremento de la prevalen - cia de diabetes y diagnóstico precoz en la población atendida en un centro de salud
}

\author{
Mạ. P. Pérez Unanua, P. Alonso Sacristán, J . C. Roiz Fernández, \\ Mạ. I. GARCía LÁZARO \\ Médicos de Familia. Centro de Salud Ciudad de los Periodistas. Madrid
}

\section{RESUMEN}

Objetivos: estimar en nuestra población el incre mento en el número de diabéticos que supone la adopción de los nuevos criterios diagnósticos (NCD). Objetivos secundarios: a) determinar la progresión a diabetes en pacientes con niveles pre vios de glucemia basal $\geq 126 \mathrm{mg} / \mathrm{dl}:$ y b) determinar diferencias en los valores de hemoglobina glicosila da $\left(\mathrm{HbA}_{1} \mathrm{c}\right)$ entre diabéticos "nuevos" y "clásicos.

Diseño: estudio observacional cohorte retrospectiva.

Material y métodos: muestreo aleatorio de his torias, población >45 años, estratificado por edad y sexo. Variables de filiación, diagnóstico diabetes, dos cifras de glucemia basal y $\mathrm{HbA}_{l} \mathrm{c}$.

Resultados: se incluyeron 803 pacientes, $224 \mathrm{mu}$ jeres $>60$ años, 27,89\%, 220 entre 45 y 59 años (27,39\%), 162 varones mayores de 60 años $(20,17 \%)$ y 197 entre 45 y 59 años $(24,53 \%)$. La prevalencia de diabetes conocida fue del 10,08\% $\pm 0,02$, el incre mento en la prevalencia tras NCD fue del 4,85\% \pm 0,015. ;Diabetes, glucemia basal alterada o un valor patológico se presentaban en un 34,98\% $\pm 0,03$ de nuestra población! Hubo 13 pacientes con glucemia previa superior a $126 \mathrm{mg} / \mathrm{dl}$ a lo largo de los 10 años de la cohorte, el tiempo medio de progresión a dia betes fue 23 meses (rango 8-48). El promedio de $H b A_{1}$ c más próxima al diagnóstico en los diabéticos clásicos fue 7,41 $\pm 0,88$ y 6,19 $\pm 0,80$ en los nuevos diabéticos, diferencia significativa $p<0,05$.
Type 2 diabetes. What do we expect after changing diagnostic criteria? Increase prevalence of diabetes and earlier diagnosis in a health care center's po pulation

\section{ABSTRACT}

Objectives: to know the increase of diabetes me llitus (DM) prevalence in our population applying ADA criteria. Secundary objective: a) time to pro gression to diabetes in patients with glucose $\geq 126$ $\mathrm{mg} / \mathrm{dl}$; and $\mathrm{b}$ ) differences between glicosilated he moglobin $\left(\mathrm{HbA}_{\mathrm{l}} \mathrm{c}\right)$ in new and classic diabetics.

Design: retrospective descriptive study.

Material and methods: aleatory sample of clini cal histories over 45 years of age, stratified by groups of age and sex. Variations: previous DM diagnosis, two fasting plasme glucose and $\mathrm{HbA}_{1} c$.

Results: the study included 803 patients, 224 women $>60$ years, $27.89 \%, 220$ between $45-59$ years $(27.39 \%), 162$ men $>60$ years $(20.17 \%)$ and 197 between $45-59$ years $(24.53 \%)$. The pre valence of $D M$ was $10.08 \% \pm 0.02$, additional in crease of prevalence with ADA criteria was $4.85 \% \pm 0.015$. Prevalence of diabetes, impaired glucose tolerance, impaired fasting glucose was $34.98 \% \pm 0.03$. There were 13 patients with glu cose $>126 \mathrm{mg} / \mathrm{dl}$, time to progression was 23 months (range 8-48). The mean of $\mathrm{HbA}_{1} \mathrm{c}$ close to diagnostic in classic diabetics was $7.41 \pm 0.88$ and $6.19 \pm 0.80$ in new diabetics, significant dif ference $p<0.05$.

Conclusions: the application of the new ADA criteria increases significantly the prevalence of diabetes and active intervention is needed to reduce

Aceptación: 17-01-02 
Conclusiones: la adopción de los NCD supone el compromiso de asumir el diagnóstico y ofrecer una intervención eficaz con el objetivo de reducir las complicaciones de la diabetes, lo que supondrá un gran esfuerzo.

Palabras clave: Diabetes. Criterios. Diagnósti co. Hemoglobina glicosilada. risk for developing macrovascular disease. It means harder work.

Key words: Diabetes. Criteria. Diagnosis. Gli cosilated hemoglobin.

\section{INTRODUCCIÓN}

La diabetes mellitus (DM) es una enfermedad crónica caracterizada por hiperglucemia y alteración del metabolismo de la glucosa. Está causada por una disminución absoluta o relativa de la secreción y/o actividad de la insulina. Se asocia a lesiones microvasculares específicas (retinopatía y nefropatía), enfermedad macrovascular por aterogénesis acelerada y otras complicaciones (embarazo de alto riesgo y mayor susceptibilidad a las infecciones).

La DM es un problema de salud de gran importancia en nuestro medio. En estudios epidemiológicos realizados en España durante los últimos diez años se estima una prevalencia para la DM tipo 2 "conocida" que oscila entre un $2,8-6,8 \%$ y un 1,8 $3,7 \%$ para la DM "desconocida" . Según se desprende de estos estudios, aproximadamente la mitad de las personas con DM no han sido diagnosticadas y el riesgo de presentar complicaciones metadiabéticas en el momento del diagnóstico es muy importante.

Los criterios diagnósticos de DM fueron revisados en 1997 por la Asociación Americana de Diabetes $(\mathrm{ADA})^{2}$. La adopción de los nuevos criterios diagnósticos (NCD) ha pretendido: a) eliminar la discrepancia pronóstica existente entre el grado de hiperglucemia reflejado por una glucemia basal (GB) $\geq 140 \mathrm{mg} / \mathrm{dl}$ y la glucemia 2 horas post sobrecarga oral de glucosa con 75 gr (SOG2h) $\geq 200 \mathrm{mg} / \mathrm{dl}$; y b) promover como test diagnóstico preferente de DM valores de $\mathrm{GB} \geq 126 \mathrm{mg} / \mathrm{dl}$. La "universalidad" (fácil y accesible) de la determinación de glucemia capilar compensará la disminución de su sensibilidad como prueba diagnóstica, si la comparamos con la SOG, y permitirá diagnosticar a un mayor número de diabéticos "desconocidos",3,4.

En septiembre de 1999, la Federación Europea de Diabetes publicó una Guía de Trabajo para la DM tipo $2^{5}$. Las principales aportaciones son el nuevo algoritmo diagnóstico y el manejo de la clasificación y de los objetivos del tratamiento en función del riesgo cardiovascular de los pacientes (Tabla I). Los nuevos criterios diagnósticos europeos de DM son en pacientes sintomáticos, con glucosuria o hiper-

\section{Tabla I}

CLASIFICACIÓN Y MANEJO DE LOS ESTADOS HIPERGLUCÉMICOS

Sintomático (con confirmación analítica): Diabetes Con riesgo de daño arterial y microvascular: Diabetes Riesgo de daño arterial por hiperglucemia y de progresión a diabetes:

Intolerancia a la glucosa

Glucemia basal alterada

glucemia casual, si la $\mathrm{GB} \geq 200 \mathrm{mg} / \mathrm{dl}$ (confirmado en 2 ocasiones) es diagnóstico de DM, si la GB $\geq 100 \mathrm{mg} / \mathrm{dl}$ hay que determinar glucemia en plasma venoso y se acepta como punto de corte diagnóstico valores de GB >125 (confirmado en 2 ocasiones) pero se establece una clara indicación para la realización del test de tolerancia oral a la glucosa cuando la glucemia en ayunas está entre 110 y 125 mg/dl. Así mismo se recomienda la revisión anual de factores de riesgo cardiovascular en todos los pacientes con GB >90 mg/dl (Tabla II).

\section{Tabla II \\ ALGORITMO DIAGNÓSTICO DE LA FEDERACIÓN EUROPEA DE DIABETES}

Si existen síntomas, glucosuria o hiperglucemia casual: Determinación glucemia al azar

$\mathrm{Si} \geq 200 \mathrm{mg} / \mathrm{dl}$ : Diabetes

$\mathrm{Si} \geq 100 \mathrm{mg} / \mathrm{dl}$, pasar al siguiente punto.

Glucemia al azar o cribado $\geq 100 \mathrm{mg} / \mathrm{dl}$ :

Determinar glucemia basal

$\mathrm{Si}>125 \mathrm{mg} / \mathrm{dl}$, repetir y confirmar: Diabetes

Si $\geq 110 \mathrm{mg} / \mathrm{dl}$, realizar SOG

$\mathrm{Si}>90 \mathrm{mg} / \mathrm{dl}$, considerar anualmente revisar FRCV

SOG

Si 2 horas $\geq 200 \mathrm{mg} / \mathrm{dl}$ : DIABETES

Si 2 horas $<200 \mathrm{mg} / \mathrm{dl}$ y $\geq 140 \mathrm{mg} / \mathrm{dl}$ : Intolerancia a la glucosa.

Si glucemia basal $\geq 110 \mathrm{mg} / \mathrm{dl}$ y SOG 2 horas $<140$ $\mathrm{mg} / \mathrm{dl}$ : Glucemia basal alterada 
El trastorno metabólico que refleja la alteración en la secreción de la insulina (SOG) se produce antes que la elevación de la GB (insulinrresistencia) ${ }^{6}$. La determinación exclusiva de la GB puede dejar sin diagnosticar a un grupo importante de pacientes jóvenes con alto riesgo de desarrollar DM y susceptibles de intervención precoz para intentar reducir la incidencia de complicaciones y éste es el motivo por el cual en el nuevo consenso europeo se sigue proponiendo la realización de la SOG para el diagnóstico de diabetes en pacientes de riesgo (diabetes gestacional previa, antecedentes familiares de diabetes, glucemia basal alterada y presencia de otros factores de riesgo cardiovascular elevados). Esta discrepancia diagnóstica disminuye en pacientes obesos y en ancianos y no se han encontrado diferencias en cuanto al sexo.

Desde su publicación han aparecido numerosos estudios que comparan la prevalencia de DM según los NCD y los antiguos criterios de la OMS $(1985)^{3,7}$. Hemos analizado los datos disponibles de nuestra población para determinar la prevalencia de diabetes y el incremento en el número de pacientes diagnosticados al adoptar los NCD.

El papel de la hemoglobina glicosilada $\left(\mathrm{HbA}_{1} \mathrm{c}\right)$ resulta de gran utilidad en la monitorización del tratamiento de la DM y su papel en el diagnóstico de la DM ha sido objeto de debate en numerosas ocasiones. El diagnóstico de DM debe establecerse en cifras de glucemia que se asocien con el desarrollo de complicaciones microvasculares específicas. Los productos finales de glucosilación avanzada constituyen un factor patogénico importante (aunque probablemente no único) en las complicaciones metadiabéticas. No deberíamos "etiquetar" como diabéticos a personas que a pesar de cifras de glucemia discretamente elevadas mantienen niveles de $\mathrm{HbA}_{1} \mathrm{c}$ normales ${ }^{8}$. Hemos analizado todos los valores disponibles de $\mathrm{HbA}_{1} \mathrm{c}$ más próximos al diagnóstico en pacientes diabéticos en los que se estableció el diagnóstico según los criterios clásicos y en aquellos diagnosticados según los NCD. Este análisis resulta interesante para determinar la importancia del diagnóstico precoz y la disminución de los factores de riesgo cardiovascular.

La progresión de pacientes con intolerancia a la glucosa (OMS85) que desarrollaran diabetes en un futuro, se cifra en torno al 1-5\% anual. Los pacientes con intolerancia a la glucosa presentan un riesgo más elevado para desarrollar complicaciones macrovasculares que la población sana. Al tratarse de un estudio histórico hemos querido examinar los pacientes diabéticos que previamente presentaban cifras de glucemia que hoy serían diagnósticas de DM según NCD y el tiempo de la progresión a diabetes clásica, para, de este modo, establecer el retraso en el diagnóstico y el tiempo que adelantarían el diagnóstico los NCD.

\section{OBJETIVOS}

Estimar la repercusión real en nuestra población del incremento en el número de pacientes diabéticos que supone la adopción de los NCD. Como objetivos secundarios nos hemos planteado ${ }^{1}$ : a) determinar si existen diferencias en los valores de $\mathrm{HbA}_{1} \mathrm{c}$ entre los pacientes diagnosticados de DM según los NCD u OMS; y b) determinar la progresión a DM en pacientes que ya presentaban niveles de glucemia basal superiores a $126 \mathrm{mg} / \mathrm{dl}$.

\section{MATERIAL Y MÉTODOS}

\section{Tipo de estudio}

Se diseñó un estudio observacional del tipo cohorte retrospectiva, realizando un muestreo aleatorio de historias clínicas estratificado y proporcional según edad y sexo. Se analizaron los datos reflejados en la historia clínica desde que se inauguró el centro de salud en 1990 hasta mayo de 2000.

\section{Marco poblacional}

El Centro de Salud Ciudad de los Periodistas atiende a una población urbana de 33.885 habitantes, de clase social media-alta.

\section{Diseño}

El estudio se realizó sobre la población mayor de 45 años porque es la edad a partir de la cual recomienda la ADA la realización de cribado sistemático para el diagnóstico de DM mediante la GB, recomendación adoptada por la semFYC en 1998. Fueron incluidos en el estudio todos aquellos pacientes que tenían historia clínica abierta y al menos una determinación de glucemia registrada en los últimos 10 años.

El muestreo inicial se hizo a partir de los listados de tarjeta sanitaria por tabla de números aleatorios. El tamaño de la muestra se calculó según la fórmula de $n$ $=1,96 \times \mathrm{p}(1-\mathrm{p}) / \mathrm{d}^{2}$, siendo el intervalo de confianza del $95 \%$, la precisión del $3 \%$ y probabilidad desconocida en el caso más desfavorable posible $(\mathrm{p}=0,5)$. La estratificación se realizó teniendo en cuenta los porcentajes de edad y sexo de nuestra población. El número de pacientes seleccionado fue 1.067.

\section{Variables}

Se recogieron variables de filiación (número de historia clínica, iniciales del paciente, edad y sexo); 
diagnóstico previo de diabetes (dato referido en la historia clínica, tratamiento antidiabético, GB $\geq 140 \mathrm{mg} / \mathrm{dl}$ (x2), SOG2h $\geq 200 \mathrm{mg} / \mathrm{dl}$ ); las dos cifras más elevadas de GB de cada paciente en $\mathrm{mg} /$ dl y su fecha correspondiente y cifra de $\mathrm{HbA}_{1} \mathrm{c}$ más próxima al diagnóstico.

\section{Análisis estadístico}

Se establecieron cinco grupos para la clasificación de los pacientes según sus glucemias y se determinó la prevalencia de cada uno de ellos:

1 . DM conocida o GB $\geq 140 \mathrm{mg} / \mathrm{dl}$ en dos ocasiones o $\mathrm{SOG} 2 \mathrm{~h} \geq 200 \mathrm{mg} / \mathrm{dl}$.

2. DM diagnosticada según $\mathrm{NCD}$ ( GB $\geq 126$ $\mathrm{mg} / \mathrm{dl}$ en dos ocasiones).

3. Glucemia basal alterada (GBA) (GB $\geq 110$ $125 \mathrm{mg} / \mathrm{dl}$ en dos ocasiones).

4. Normales.

5. Pacientes con un único valor alterado no clasificados en ninguno de los grupos anteriores.

Se determinó el tiempo medio de progresión a DM en aquellos pacientes que previamente presentaban cifras de GB superiores a $126 \mathrm{mg} / \mathrm{dl}$ y que habían desarrollado diabetes (GB $\geq 140 \mathrm{mg} / \mathrm{dl}$ x 2) a lo largo de los diez años del estudio histórico con resultados interesantes que comentaremos más adelante.

Así mismo se analizó si existían diferencias entre los valores promedio de $\mathrm{HbA}_{1} \mathrm{c}$ en el grupo 1 de pacientes con diabetes conocida y en el grupo 2 de pacientes con diabetes según NCD.

\section{RESULTADOS}

El número final de pacientes estudiados fue de 803 , lo que supone una pérdida del $23,80 \%$ de las historias. La principal causa de no inclusión en el estudio fue la ausencia de datos analíticos en la historia clínica. Los grupos estratificados por edad y sexo quedaron del siguiente modo: 224 mujeres mayores de 60 años $(27,89 \%), 220$ mujeres entre 45 y 59 años (27,39\%), 162 varones mayores de 60 años $(20,17 \%)$ y 197 varones entre 45 y 59 años $(24,53 \%)$.

El número de diabéticos que ya estaban diagnosticados según los criterios clásicos fue de 81 pacientes, lo que supone una prevalencia de diabetes conocida en nuestra población del 10,08\% (IC 95\%, 10,06-10,1). El número de nuevos pacientes diabéticos diagnosticados tras la adopción de las actuales recomendaciones de la ADA y de la FED fue de 39, lo que supone un incremento en la prevalencia de DM de un 4,85\% (IC 95\% 4,835-4,865).

Aplicando los NCD, analizamos el número de pacientes que presentaban una alteración de la glu- cemia basal, y que serían susceptibles de un seguimiento cercano con revisión anual de todos los factores de riesgo vascular. El número de pacientes con alteración de la glucemia basal, confirmada en dos ocasiones (110-125 mg/dl) fue de 83, lo que supone una prevalencia del 10,33\% (IC 95\% 10,31-10,35). Se determinó también como dato orientativo aquellos pacientes que presentaban un único valor de glucemia alterado resultando 73 pacientes $(9,09 \%$, IC 95\% 9,07-9,11). De este modo, si sumamos estos porcentajes, nos encontramos con que un 34,98\% (IC 95\% 34,95-35,01) de la población de nuestra zona básica de salud presenta una alteración en los niveles de glucemia. En cuanto a la edad vemos que existe un incremento progresivo en la prevalencia de diabetes en los grupos de edad avanzados. No existen diferencias significativas en cuanto al sexo. Estos datos se muestran desglosados por grupos de edad y sexo según muestran las figuras 1 y 2 .

Al tratarse de un estudio de estas características, hemos podido analizar datos acerca del periodo de tiempo transcurrido desde la elevación de la glucemia basal (ADA, >126 mg/dl) hasta el diagnóstico de diabetes clásica (OMS, >140 mg/dl), calculando el tiempo promedio que determinó la progresión a diabetes en este grupo de pacientes y determinando el tiempo que se podía haber adelantado el diagnóstico y la intervención. Hubo 13 pacientes diabéticos $(16,05 \%$, IC 95\% 15,99-16,11) que ya presentaban niveles de glucemia superiores a $126 \mathrm{mg} / \mathrm{dl} \mathrm{a}$ lo largo de los diez años de la cohorte histórica. De ellos, 6 pacientes $(46,15 \%$, IC 95\% 45,87-46,43) desarrollaron diabetes en el primer año tras la elevación de la GB, 3 pacientes $(23,07 \%$, IC 95\% 22,84-23,3) en los dos años siguientes, 3 pacientes $(23,07 \%$, IC $95 \% 22,84-23,3)$ a lo largo del tercer año y 1 paciente $(7,69 \%$, IC 95\% 7,54-7,84) a los cuatro años. El tiempo medio de progresión a diabetes fue de 23 meses (rango 8-48 meses).

El examen de la distribución de los valores de $\mathrm{HbA}_{1} \mathrm{c}$ de los pacientes en los que se estableció el diagnóstico de diabetes resultó como sigue: de los 86 pacientes con DM conocida, $48(55,81 \%)$ disponían registrados valores de $\mathrm{HbA}_{1} \mathrm{c}$ en el momento del diagnóstico. En estos pacientes el valor medio de $\mathrm{HbA}_{1} \mathrm{c}$ más próxima al diagnóstico fue de $7,41 \%$ $\pm 0,88$. De los 39 pacientes diabéticos según los NCD únicamente había $8(20,51 \%)$ que tenían registrado en la historia los niveles de $\mathrm{HbA}_{1} \mathrm{c}$. En estos pacientes el valor medio fue de $6,19 \pm 0,80$. Esta diferencia entre un grupo y otro resultó estadísticamente significativa $(\mathrm{p}<0,05)$. Estos datos son importantes ya que el diagnóstico de diabetes es controvertido en el caso de pacientes con niveles normales de $\mathrm{HbA}_{1} \mathrm{c}$ y debe establecerse en función de cifras de glucemia que se asocian con el desarrollo de complicaciones específicas. 

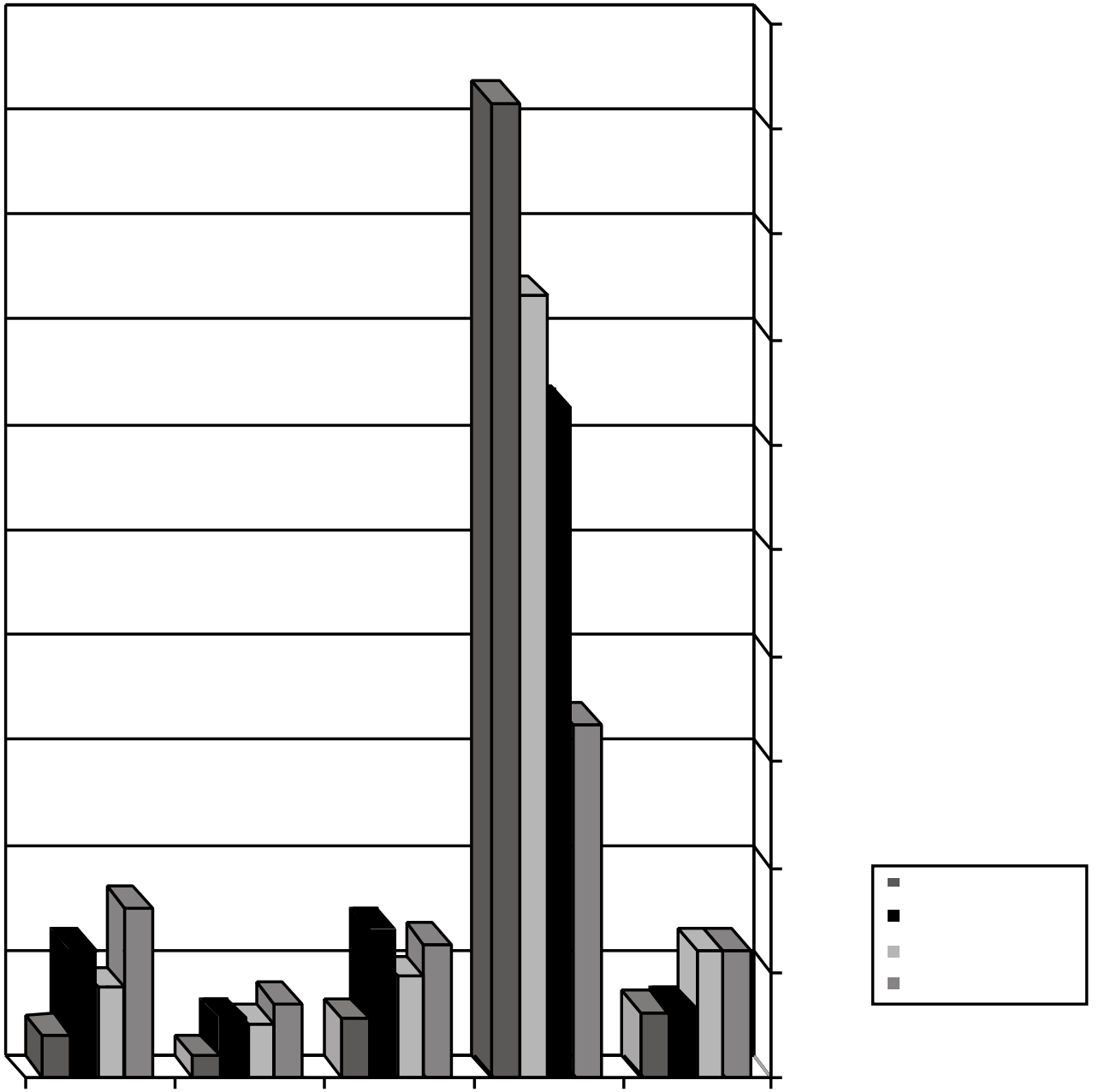

Resultados del estudio. Prevalencia de las alteraciones del metabolismo de la glucosa en nuestra población

\section{DISCUSION}

Desde su publicación han aparecido numerosos estudios que comparan la prevalencia de DN según los NCD (ADA, 1997) y los antiguos criterios clásicos de la OMS (1985). Los resultados, hasta ahora reflejan una baja concordancia entre ambos tests diagnósticos, con diferencias significativas en las prevalencias de DM identificadas según uno u otro criterio. No obstante los resultados son dispares, según se desprende del metaanálisis europeo publicado en 1998, la adopción de los NCD puede suponer desde un descenso del $4 \%$ a un incremento del $13,2 \%$ en la prevalencia de DM ${ }^{9}$. El NHANES III refleja una prevalencia de DM en EE.UU. del $7,9 \%$, y una prevalencia de DM no diagnosticada del 4,4\% según los NCD y del 6,4\% según los criterios de la OMS ${ }^{9}$.
Hemos querido exponer nuestros datos al hilo de las numerosas publicaciones que han aparecido en la prensa médica en los últimos años desde la publicación de los NCD en 1997. En nuestro estudio la prevalencia de la DM2 según criterios clásicos $(10,08 \%$, IC $10,04-10,12)$ es similar a la que publican Pulgar et al. ${ }^{11}$ aunque elevada si la comparamos los datos de población de otras publicaciones ${ }^{10}$. Hay que tener en cuenta que este estudio se ha realizado sobre población demandante con al menos una analítica registrada en la historia clínica, no sobre población general y ésta puede ser la causa de estas diferencias y de la elevada prevalencia que encontramos. Nuestros resultados muestran un incremento en la prevalencia de DM de un $4,85 \%$ (IC: 4,55-5,15), cifra más elevada que en otros estudios españoles ${ }^{12-14}$ aunque similar a la obtenida por los americanos en el NHANES III. 


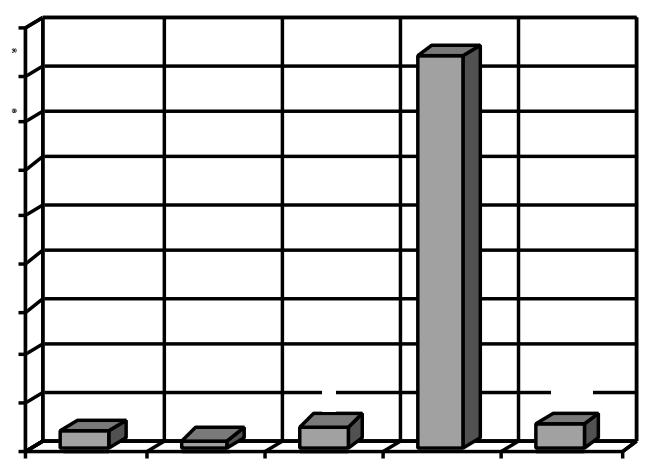

$\square$

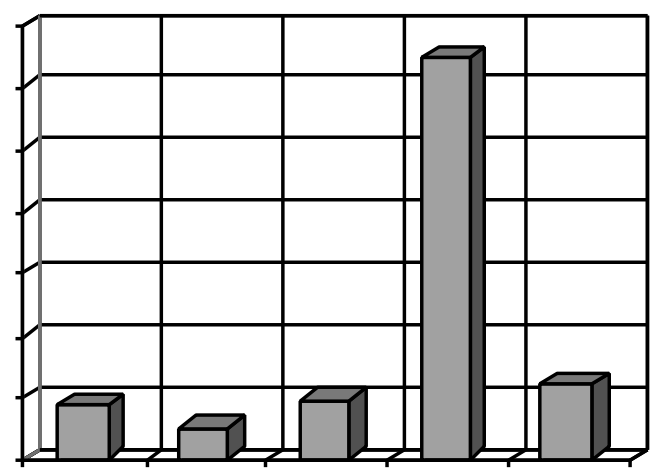

$\square$

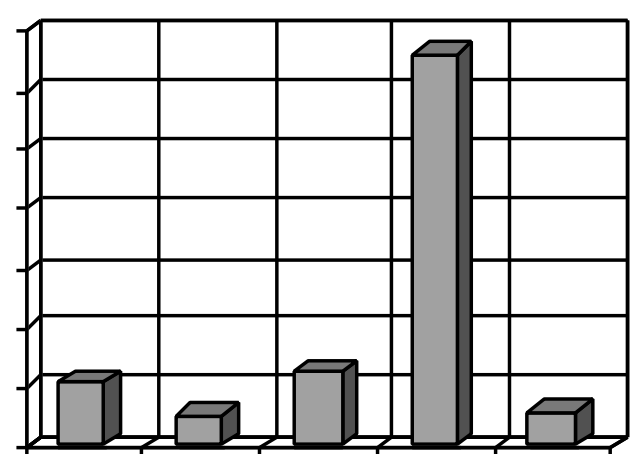

口

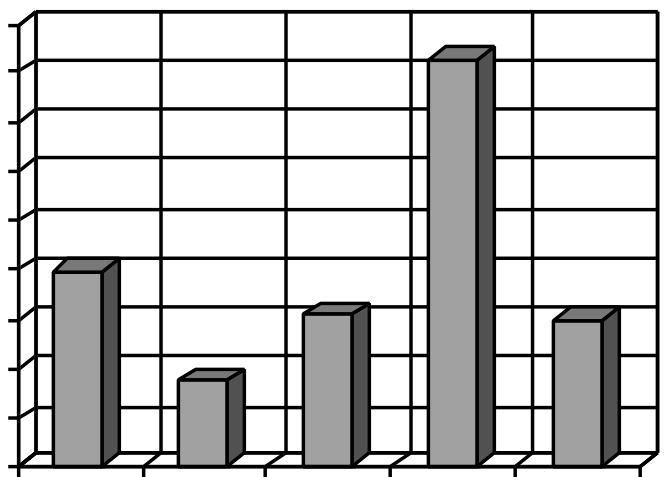

口
El estudio está realizado sobre población mayor de 45 años, de este modo la mayoría de los pacientes van a ser diabéticos tipo 2 , el porcentaje de diabetes tipo 1 es mínimo y porque es la edad a partir de la cual recomienda la ADA la realización de cribado sistemático para el diagnóstico de diabetes mediante la GB, recomendación adoptada por la semFYC en 1998.

En cuanto al tamaño de la muestra podía haber sido inferior si se hubiera calculado en función de la prevalencia esperada de diabetes en la población española. De haberlo hecho así, los resultados probablemente no hubieran sido los mismos pues nos hemos encontrado con prevalencias más elevadas que las que aparecen en los trabajos publicados.

Para establecer el diagnóstico final (DM, GBA, NORMAL) se ha requerido en todos los casos la confirmación de los valores en dos determinaciones. Esto es importante ya que en numerosos tra- bajos han establecido el diagnóstico de diabetes teniendo en cuenta una única determinación, la de la SOG que realizaron para el estudio. Este proceder no es correcto, dando lugar a clasificaciones erróneas de algunos individuos, no así en nuestro trabajo.

La prevalencia de diabetes en nuestra comunidad aumenta con la edad. En cuanto al sexo, el porcentaje de diabéticos fue mayor en el grupo de varones que en mujeres, datos que concuerdan con la literatura.

Dada la importancia de la glicosilación excesiva de las proteínas en el origen y desarrollo de las complicaciones diabéticas, algunos autores sugieren una estrategia diagnóstica alternativa que combine la determinación de glucemia basal y la $\mathrm{HbA}_{1} \mathrm{c}$. La diferencia significativa de los niveles de $\mathrm{HbA}_{1} \mathrm{c}$ que encontramos entre el grupo de pacientes diabéticos clásicos (GB $\geq 140 \mathrm{mg} / \mathrm{dl}$, 
sin tener en cuenta la SOG) y los diabéticos diagnosticados según los NCD (GB $\geq 126 \mathrm{mg} / \mathrm{dl}$ ) indicaría que estos "nuevos diabéticos" son menos diabéticos, ya que lógicamente el grado de hiperglucemia es menor y el diagnóstico se establece antes. Después del UKPDS, ha quedado claro que la disminución de la hemoglobina glicosilada mediante una intensificación del tratamiento y el control global de todos los factores de riesgo cardiovascular es capaz de reducir el número y la gravedad de las complicaciones, una vez realizado el diagnóstico ${ }^{15-17}$. A estos pacientes de riesgo se les identificará y habrá que intervenir de forma adecuada con dieta y ejercicio para disminuir la morbimortalidad cardiovascular y otras complicaciones derivadas de la diabetes que al fin y al cabo es de lo que se trata. El problema radica en que las determinaciones de $\mathrm{HbA}_{1} \mathrm{c}$ no están estandarizadas por lo que resulta difícil su aplicación. En nuestro estudio no todos los pacientes presentaban esta determinación lo que podría falsear los datos obtenidos.

Nuestro estudio aporta datos del tiempo de progresión de la GB desde que se diagnostica por primera vez una elevación por encima de $126 \mathrm{mg} / \mathrm{dl}$ hasta llegar a $140 \mathrm{mg} / \mathrm{dl}$, es decir el tiempo que los NCD “adelantarían” el diagnóstico de diabetes. Este dato es muy importante de cara a la intervención precoz sobre estos pacientes para evitar, al igual que hemos comentado antes, el desarrollo de complicaciones y disminuir el riesgo cardiovascular. Dinneen et al. publicaron un estudio sobre 7.567 individuos no diabéticos mayores de 40 años seguidos durante 9 años, demostrando que la aplicación de los criterios de la ADA adelantaría en 7 años el diagnóstico ${ }^{18}$. La aplicación exclusiva de la glucemia basal como herramienta diagnóstica dejaría sin diagnosticar a algunos pacientes diabéticos, pero menos de los que se detectarán al disminuir el punto de corte diagnóstico a 126.

Existen categorías intermedias de la alteración del metabolismo glucídico: la intolerancia a la glucosa (IG) y una nueva entidad la glucemia basal alterada. La prevalencia de GBA es mayor que la
DM en la población general ${ }^{19}$. La relación entre IG y complicaciones macrovasculares está bien establecida. La prevención primaria no farmacológica (dieta y ejercicio físico) ha demostrado ser eficaz para reducir la incidencia de DM en este grupo de pacientes ${ }^{20}$. No existen evidencias todavía para el grupo de pacientes con GBA, probablemente los resultados fueran similares, aunque se necesitará mucho trabajo para abarcar correctamente a tanta población.

Por último es importante realizar estudios en términos económicos que confirmen a largo plazo los beneficios del diagnóstico precoz de diabetes y ver si todo este esfuerzo se compensa con la reducción de las complicaciones micro y macrovasculares de la diabetes en estos pacientes. ¿Qué significado real tendrá tener "más" diabéticos a los que recomendar hábitos de vida saludables? ¿Disminuirá la incidencia de cardiopatía isquémica al tratar más agresivamente el resto de factores de riesgo cardiovascular en este grupo de pacientes? Posiblemente ninguno de los componentes de la atención médica tenga mayor importancia para el posterior cuidado del paciente que el establecimiento del diagnóstico. Sobre esta base se toman decisiones respecto al tratamiento, pronóstico y utilización de recursos sanitarios. Con frecuencia la separación entre hallazgos normales y anormales es arbitraria y se basa en procedimientos estadísticos; con el diagnóstico de la enfermedad, el tratamiento podría prevenir o retrasar el inicio de acontecimientos adversos posteriores $^{21}$.

\section{CORRESPONDENCIA:}

$M^{a}$. Paz Pérez Unanua

Plaza Peña Horcajo, 11, portal B, $5^{\circ} \mathrm{B}$

28035 Madrid

Telf.: 917370175

e-mail: mpperezunanua@ hotmail.com

\section{Bibliografía}

1. Diabetes tipo 2 en España. La magnitud del problema: ¿ Cuántas personas con diabetes hay en España? [Editorial]. Educación Diabetológica Profesional 1999; 10: 4-5.

2. ADA. Report Of The Expert Committee On The Diagnosis And Classification Of Diabetes Melllitus. Diabetes Care 1997; 20: 1183-97.

3. Wahl PW, Savage PJ, Psaty BM, Orchard TJ, Robbins JA, Tracy RP. Diabetes in older adults: comparison of 1997 Ame- rican Diabetes Association classification of diabetes mellitus with 1985 WHO classification. Lancet 1998; 352: 1012-5.

4. New diagnostic criteria for diabetes and mortality in older adults. [Letter]. Lancet 1999; 353: 68-70.

5. European Diabetes Policy Group 1998-1999. International Diabetes Federation, European Region. A Desktop Guide To Type 2 Diabetes Mellitus. Exp Clin Endocrinol Diabetes 1999; 107: 390-420. 
6. Meneilly GS, Elliot T. Metabolic alterations in middle aged and elderly obese patients with type 2 diabetes. Diabetes Care 1999; 22: 112-8.

7. Harris MI, Eastman RC, Cowwie CC, Flegal KM, Eberhardt MS. Comparison of diabetes diagnostic categories in the U.S. population according to 1997 American Diabetes Association and 1980-1985 World Health Organization diagnostic criteria. Diabetes Care 1997; 20: 1859-62.

8. Davidson MB, Schriger DL, Peters AL, Lorber B. Relación entre la glucosa plasmática en ayunas y la hemoglobina glucosilada. Potencial de diagnósticos falsos positivos de diabetes tipo 2 utilizando los nuevos criterios diagnósticos. JAMA 1999; 281: 1203-10. JAMA (ed. esp.) 1999; 8: 424-33.

9. DECODE Study Group. Will new diagnostic criteria for diabetes mellitus change phenotype of patients with diabetes? Reanalysis of European epidemiological data. BMJ 1998; 317: 371-5.

10. Diabetes tipo 2 en España. La magnitud del problema: ¿cúantas personas con diabetes hay en España? [Editorial]. Educación Diabetológica Profesional 1999; 10: 4-5.

11. Pulgar Suárez M, Gómez Guedes P, Aguado Díaz M, Menéndez Álvarez S, García Garaboa A, Rodríguez González I, et al. Validez de los nuevos criterios diagnósticos de la diabetes mellitus tipo 2. Impacto de su aplicación en un área de salud. Aten Primaria 2001; 27: 111-5.

12. Sánchez Sánchez RM, Escudero Martín C, Callejo Villlarrrubia B. Incremento de la prevalencia de diabetes mellitus conocida aplicando los nuevos criterios diagnósticos de la American Diabetes Association. Medifam 1999; 4: 216-21.

13. Valdivieso Mañas MC, Puertas Cabot A, Martínez López MJ, Rodriguez García A, Mañes Ridaura C, Ballester Donet A, et al. Estudio de la repercusión de la aplicación de los nuevos criterios de la ADA sobre la prevalencia de diabetes mellitus en pacientes hipertensos. Medifam 1999; 4: 227-30.

14. Baena Díez JM, Oller Colom M, Martín Peñacoba R, Nicolau Sabaté M, Altes Boronat A, Iglesias Serrano C. Impacto de los nuevos criterios diagnósticos propuestos por la Asociacion Americana de Diabetes (ADA-97) sobre la prevalencia diagnóstica de la diabetes mellitus tipo 2. Aten Primaria 1999; 24: 97-100.

15. Peters AL, Schringer DL. The new diagnostic criteria for diabetes: the impact on management of diabetes and macrovascular risk factors. Am J Med 1998; 105(1A): 15S-19S.

16. American Diabetes Association. Implications of the United Kingdom Prospective Diabetes Study. Diabetes Care 1999; 22 (Supl. 1): 27-31.

17. UK Prospective Diabetes Study Group. Tight blood presure controland risk of macrovascular complications in type 2 diabetes (UKPDS 38). BMJ 1998; 317: 703-13.

18. Dinneen SF, Maldonado III D, Leibson CL, Klee GG, Li H, Melton III LJ, et al. Effects of changing diagnostic criteria on the risk of developing diabetes. Diabetes Care 1998; 21: 1408-13.

19. Costa B, Martín F, Donado A, Parera F, Piñol JL, Basora J, et al. Diabetes ignorada y otras alteraciones del metabolismo glucídico en la población española de alto riesgo. El estudio ITG. Med Clin (Barc) 2000; 114: 601-8.

20. Eriksson J, Lindström J, Valle T, Aunola S, Hämäläinen H, Ilane-Parika P, et al. Prevention of type 2 diabetes in subjects with impaired glucose tolerance: the Diabetes Prevention Study (DPS). Finland, Diabetologia 1999; 42: 793-801.

21. Vinicor F. ¿Cuándo la diabetes es diabetes? Jama (ed. esp.) 1999; 8: 409-12. 